Original research article

\title{
Quantification of uPA in breast tumour tissue extracts by microarray immunoassay: Comparison with ELISA technology
}

\author{
Liu Shi ${ }^{\mathrm{a}}$, Thomas Gehin ${ }^{\mathrm{a}}$, Yann Chevolot ${ }^{\mathrm{a}}$, William Jacot ${ }^{\mathrm{b}}$, Pierre-Jean Lamy ${ }^{\mathrm{c}, \mathrm{d}}$, \\ Emmanuelle Laurenceau ${ }^{\mathrm{a}, *}$ \\ a Lyon University, Lyon Institute of Nanotechnology INL-UMR5270, CNRS, Ecole Centrale de Lyon, Ecully, France \\ ${ }^{\mathrm{b}}$ Montpellier Cancer Institute Val d'Aurelle, Medical Oncoly Department, Montpellier, France \\ ${ }^{\mathrm{c}}$ Institut Médical d'Analyse Génomique-Labosud, Montpellier, France \\ d Montpellier Cancer Institute Val d'Aurelle, Translational research unit and Biological Ressources Center, Montpellier, France
}

\section{A R T I C L E I N F O}

\section{Article history:}

Received 3 August 2017

Received in revised form 17 November 2017

Accepted 11 January 2018

Available online 3 February 2018

\section{Keywords:}

\section{Breast cancer}

uPA quantification

Nano level techniques

Immunological approaches

Antibody microarray

\section{A B S T R A C T}

The urokinase-type plasminogen activator (UPA) and PA inhibitor 1 (PAI-1) play important roles in breast cancer metastasis through cell migration and invasion. They are clinically applicable prognostic and predictive markers. High levels of uPA and PAI-1 are associated with high risk of recurrence and adjuvant chemotherapy provides substantial benefit for this breast cancer population. The current sole validated method for quantifying uPA level in breast tumour tissue is ELISA assay. It requires 50-300 mg of fresh or frozen tissue, which is the main limitation for routine use. In this study, we evaluated the performances of customized antibody microarray to quantify uPA concentration from reduced extraction solution of breast tumour tissue and compared it with standard ELISA kit. We firstly optimized the elaboration of customized antibody microarray in order to sensitively detect and quantify uPA standard solutions. In the best conditions, we analysed uPA concentration in 16 cytosolic extracts from breast tumour tissue. Results showed that our customized antibody microarray could correctly quantify uPA concentration while consuming 100 times less volume of tumour tissue extraction solution than ELISA. Our antibody microarray is a powerful and promising tool for the miniaturization of the immunoassay quantification of uPA from breast tumour tissue extracts.

(C) 2018 Faculty of Health and Social Sciences, University of South Bohemia in Ceske Budejovice. Published by Elsevier Sp. z o.o. All rights reserved.

\section{Introduction}

Urokinase-type plasminogen activator (uPA), uPA receptor (uPAR) and uPA inhibitors 1 and 2 (PAI-1 and PAI-2) play important roles in physiologic processes such as detachment and cell migration, but also in pathologic processes such as tumour growth, invasion and metastasis (Kwaan et al., 2013; McMahon and Kwaan, 2008). They are involved in many human cancers, including breast, prostate, lung, brain, ovary, etc. Due to their involvement in cancer-related function, various retrospective and prospective studies showed that uPA and PAI- 1 are good prognostic and predictive biomarkers in breast cancer. Low levels of uPA $(\leq 3 \mathrm{ng} / \mathrm{mg}$ of total protein) and PAI-1 ( $\leq 14 \mathrm{ng} / \mathrm{mg}$ of total protein)

\footnotetext{
* Author for correspondence: Lyon University, Lyon Institute of Nanotechnology INL-UMR5270, CNRS, Ecole Centrale de Lyon, 36 Avenue Guy de Collongue, 69134 Écully Cedex, France.

E-mail address: emmanuelle.laurenceau@ec-lyon.fr (E. Laurenceau).
}

are associated with low risk of recurrence and may have few or no benefit from adjuvant chemotherapy. On the contrary, high levels of uPA and PAI- 1 are correlated with high risk of recurrence and adjuvant chemotherapy provides substantial benefit for these breast cancer patients (Harbeck et al., 2002, 2013; Janicke et al., 2001; Look et al., 2002). uPA and PAI-1 have the highest level-ofevidence (LOE-1) for providing the prognostic and predictive value for node-negative breast cancer patients (Duffy et al., 2014; Harris et al., 2016).

ELISA is the only method which is recommended by the American Society of Clinical Oncology (ASCO) to determine uPA and PAI-1 concentrations in cytosolic extracts from fresh or frozen breast tumour tissue. The commercially available ELISA test (Femtelle) was developed by American Diagnostica (Sekisui Diagnostics) and it requires $50-300 \mathrm{mg}$ of fresh or frozen breast tumour tissue. However, the main source of patient's sample worldwide is formalin-fixed paraffin-embedded (FFPE) tissue (Becker et al., 2007). Furthermore, the need for large quantity of 
tissue requires surgical biopsy or vacuum-assisted core biopsy with an 8-gauge needle (Wilson and Kavia, 2009) and precludes the use of 14-gauge needle-core biopsies that are more common in clinical practice (Schueller et al., 2008). Thus, requirement of large quantity of fresh tissue becomes the main limitation of ELISA assays.

Protein microarrays have several advantages compared with traditional ELISA including high sensitivity and tiny volume sample consumption (Cretich et al., 2014; Sutandy et al., 2013). Antibody microarray was used to quantify uPA and PAI- 1 in cytosolic extracts from breast cancer tissues while the performance need to be improved (Weissenstein et al., 2006). Reverse-phase protein arrays (RPPA) was also used and it could quantify total protein content by using Sypro-Ruby protein stain. One study used RPPA to quantify uPA from FFPE breast cancer tissues and found that the arrays could distinguish positive and negative samples, while only two samples (one positive and one negative) were involved in this study (Malinowsky et al., 2010). Moreover, the efficiency of protein microarray still remains challenging because it is influenced by various factors, including surface chemistry, $\mathrm{pH}$ of spotting buffer and concentration of immobilized proteins (Balboni et al., 2008; Hu et al., 2012; Shukla et al., 2007; Yang et al., 2012b). This study therefore aims to develop a sensitive antibody microarray to quantify uPA in cytosolic extracts from frozen breast tumour tissues. We firstly optimized conditions for antibody microarray (surface chemistry, antibody concentrations). Then using the best conditions, we quantified uPA concentration in 16 cytosolic extracts from frozen breast tumour tissues and compared results with Femtelle ELISA kit.

\section{Materials and methods}

\section{Materials}

Glass and all chemicals were commercial available unless otherwise stated. Borosilicate flat glass slides $(76 \times 26 \times 1 \mathrm{~cm})$ were purchased from Schott (Mainz, Germany). $0.01 \mathrm{M}$ phosphatebuffered saline (PBS, pH 7.4), N-Hydroxysuccinimide (NHS), $N, N$-diisopropylcarbodiimide (DIC), tetrahydrofuran (THF) (purum grade), Dimethyl sulfoxide (DMSO, anhydrous, 99.9\%), 2-(N-morpholino) ethanesulfonic acid (MES), (3-glycidoxypropyl) dimethylethoxysilane (APDMES) and maleic anhydride-alt-methyl vinyl ether (MAMVE, Mw $=216,000 \mathrm{~g} / \mathrm{mol}$ ) were all obtained from Sigma (St. Quentin Fallavier, France). Dextran $(\mathrm{Mw}=40,000 \mathrm{~g} / \mathrm{mol})$ and Tween 20 were purchased from Pharmacosmos and RothSochiel (Lauterbourg, France), respectively. Chitosan $(\mathrm{Mw}=470,000 \mathrm{~g} / \mathrm{mol}$, degree of deacetylation (DD) 94\%) was kindly provided by Dr. T. Delair (Laboratoire d'Ingénierie des Matériaux Polymères (IMP), CNRS-University of Lyon, France). Ultrapure water (18.2 $\mathrm{M} \Omega$ ) was delivered by an Elga water system.

Anti-uPA antibodies (mouse monoclonal) were obtained from Santa Cruz Biotech (sc-59729) and Thermo Scientific (MON U-1602); Femtelle kit was purchased from American Diagnostica Inc; F555-labeled streptavidin (S-21381) was obtained from Invitrogen. All proteins were stored as aliquot at $-20^{\circ} \mathrm{C}$ or $-80^{\circ} \mathrm{C}$ following manufacturer specifications. Bovine serum albumin (BSA) lyophilized powder was obtained from Sigma (St. Quentin Fallavier, France).

$0.01 \mathrm{M}$ 2-(N-morpholino) ethanesulfonicacid (MES) ( $\mathrm{pH} 6.2$ ) was prepared by dissolving the content of one pouch in to 11 ultrapure water and adjust $\mathrm{pH}$ up to 6.2. 0.01 M PBS or PBS $1 \mathrm{X}(\mathrm{pH}$ 7.4) was prepared by dissolving the content of one pouch of dried powder in 11 of ultrapure water. Washing buffer contained PBS $1 \mathrm{X}$ and $0.1 \%$ Tween 20 (PBS-T) at $\mathrm{pH}$ 7.4. Blocking solution was prepared by dissolving $10 \mathrm{~g}$ of BSA in $100 \mathrm{ml}$ of PBS-T $0.1 \%$.
Biological samples

Aliquots $(50 \mu \mathrm{l})$ of 16 cytosolic extracts prepared from frozen estrogen receptor-positive, HER2-negative, breast tumour tissue samples (36-320 mg) were provided by Biobank number BB-003300059 (Biological Ressources Center, Montpellier Cancer Institute [ICM] Val d'Aurelle, Montpellier, France, France). This study was reviewed and approved by the ICM Institutional Review Board. All patients gave their written, informed consent. Each cytosolic extract was previously evaluated at ICM from other aliquots for total protein content using Pierce BCA Protein Assay Reagent Kit provided by Thermo Fisher Scientific and for uPA levels using Femtelle kit. The concentration of uPA in total protein was obtained from uPA levels divided by total protein content.

\section{Surface functionalization of microstructured glass slides}

Microwells were generated on the surface of flat glass slides by photolithography (Mazurczyk et al., 2008). Microwells are $3 \mathrm{~mm}$ side length, $102 \pm 1 \mu \mathrm{m}$ depth, and spacing between each well is $4.5 \mathrm{~mm}$. The details of surface functionalization of glass slides are reported in (Yang et al., 2012b). Briefly, flat and microstructured glass slides were functionalized with 6 different chemistries: Carboxylic surface $(\mathrm{COOH})$ was obtained after hydrolysis of the tertbutyl esters from tert-butyl-11-(dimethylamino) silylundecanoate silanized surface; NHS surface was obtained from $\mathrm{N}$-hydroxysuccinimide activation of $\mathrm{COOH}$ surface; Chitosan surface was obtained by functionalization of the NHS surface with $1 \mathrm{mg} / \mathrm{ml}$ chitosan solution; APDMES surface was obtained by silanization with (3-aminopropyl) dimethylethoxysilane; MAMVE and CMD surfaces were obtained by functionalization of APDMES surface with maleic anhydride-methyl vinyl ether copolymer solution $(1 \mathrm{mg} / \mathrm{ml})$ and NHS-activated carboxymethyl dextran solution $(1 \mathrm{mg} / \mathrm{ml})$, respectively.

Evaluation of the biological activity of antibodies against UPA with the Femtelle kit

The biological activity of two capture anti-uPA antibodies was evaluated by standard ELISA method. Briefly, $100 \mu$ l captured antibodies $(0.1 \mathrm{mg} / \mathrm{ml})$ were added in each well of a 96 -wells plate (supplied by Greiner Bio One) and incubated overnight at room temperature (R.T.); then solutions were removed and washed with $200 \mu \mathrm{l}$ per well of washing buffer (PBS $1 \mathrm{X}-0.1 \%$ Triton X-100) for $3 \times 5$ min. After blocking with $1 \%(\mathrm{w} / \mathrm{v})$ BSA (dissolved in PBS-T $0.1 \%$ ) for $2 \mathrm{~h}$ at R.T., $100 \mu \mathrm{l}$ uPA standard solutions (from Femtelle kit) were added into wells and incubated for $1 \mathrm{~h}$ at R.T., then solutions were removed and washed for $3 \times 5 \mathrm{~min}$ with washing buffer. Then wells were incubated with enzyme conjugate (from Femtelle kit: $1 \mu \mathrm{l}$ enzyme conjugate in $1 \mathrm{ml}$ enzyme conjugate diluent) for $1 \mathrm{~h}$ at R.T. After washing, $100 \mu \mathrm{l}$ substrate solution (from Femtelle kit) and stopping solution $\left(0.5 \mathrm{~N} \mathrm{H}_{2} \mathrm{SO}_{4}\right)$ were added sequentially. The solutions' colour turned yellow and then the absorbance was measured at $450 \mathrm{~nm}$ within $30 \mathrm{~min}$. The Femtelle ELISA was conducted according to the protocol provided by the kit.

\section{Optimization of antibody microarray conditions}

Both anti-uPA antibodies were spotted (sciFLEX-ARRAYER S3, Scienion, Germany) in PBS $1 \mathrm{X}$ buffer ( $\mathrm{pH}$ 7.4) on 6 chemically functionalized microstructured glass slides at different concentrations: $0.33 \mu \mathrm{M}, 0.67 \mu \mathrm{M}$ and $2.5 \mu \mathrm{M}$ for anti-uPA antibody from Santa Cruz Biotech, $3 \mu \mathrm{M}$ and $6.6 \mu \mathrm{M}$ for anti-uPA antibody from Thermo Scientific, as described in Fig. 1. Each concentration was spotted in 6 replications in each microwell. PBS $1 \mathrm{X}$ and streptavidin-F555 $(0.01 \mathrm{mg} / \mathrm{ml})$ were spotted as negative and 


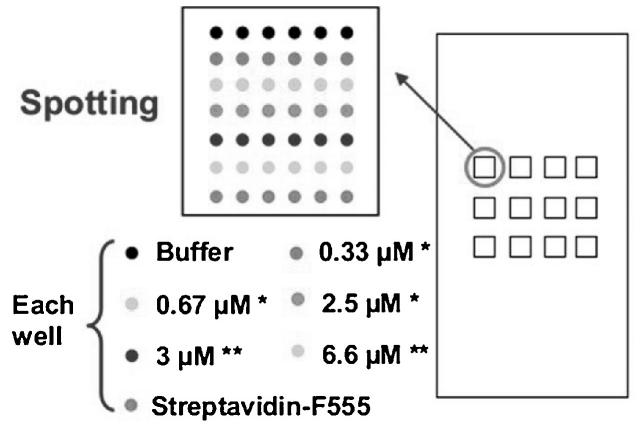

$1^{\text {st }}$ incubation

uPA (ng /ml):

$0,0.2,0.5,1,1.5,2$

$2^{\text {nd }}$ incubation

Anti-uPA antibody-biotin:

- Non-diluted: 6 wells

$-1 / 2$ diluted: 6 wells

\section{Experimental scheme}

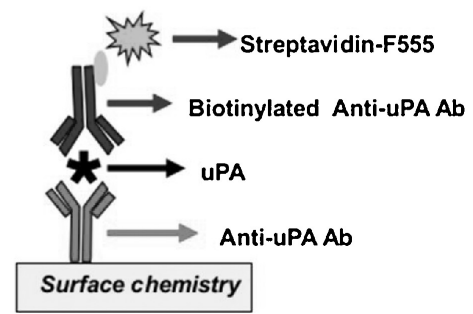

Fig. 1. Scheme of antibody microarray design to optimize experimental conditions. Spotting: anti-uPA antibody was spotted in PBS $1 \mathrm{X}$ at different concentrations ( $0.33 \mu \mathrm{M}$, $0.67 \mu \mathrm{M}$ and $2.5 \mu \mathrm{M}$ for antibody from Santa Cruz Biotech, and $3 \mu \mathrm{M}$ and $6.6 \mu \mathrm{M}$ for antibody from Thermo Scientific) with 6 replications for each concentration. PBS $1 \mathrm{X}$ (Buffer) and streptavidin-F555 (Strep-F555) were spotted as negative and quality controls, respectively; 1st incubation: uPA at 6 different concentrations (each concentration in duplicate); 2nd incubation was performed with non-diluted and diluted (1:2) biotinylated anti-uPA antibody; *antibody from Santa Cruz Biotech; **antibody from Thermo Scientific.

quality controls, respectively. After spotting, antibodies were allowed to react with surfaces under saturated water vapours overnight at $4{ }^{\circ} \mathrm{C}$. Then spotted slides were washed sequentially for $2 \times 5$ min with PBS 1 X, for 5 min with PBS-T (0.1\%), and blocked with $10 \% \mathrm{BSA} / \mathrm{PBS}-\mathrm{T}$ solution for $2 \mathrm{~h}$ at room temperature (R.T.) to limit unspecific adsorption, then washed for $3 \times 5$ min with PBS-T and dried by centrifugation $3 \mathrm{~min}$ at $1300 \mathrm{rpm}$.

Slides were then incubated with 6 different standard concentrations of uPA $(0 \mathrm{ng} / \mathrm{ml}, 0.2 \mathrm{ng} / \mathrm{ml}, 0.5 \mathrm{ng} / \mathrm{ml}, 1 \mathrm{ng} / \mathrm{ml}, 1.5 \mathrm{ng} / \mathrm{ml}$ and $2 \mathrm{ng} / \mathrm{ml}$ ) prepared from Femtelle kit, as shown in Fig. 1. Each standard concentration of uPA was tested in duplicate. Slides were left to react for $1 \mathrm{~h}$ at R.T. in saturated water vapours, then washed for $3 \times 5$ min with PBS-T and dried.

Then slides were incubated with non-diluted and diluted (1:2 in $4 \%$ BSA/PBS-T $0.1 \%$ ) biotinylated anti-uPA anti-body prepared from Femtelle kit, as shown in Fig. 1. The incubations were left to react for $1 \mathrm{~h}$ at R.T. in saturated water vapours, and then slides were washed for $3 \times 5$ min with PBS-T and dried. Then microwells were incubated with streptavidin-F555 $(0.01 \mathrm{mg} / \mathrm{ml}$ diluted in $1 \% \mathrm{BSA} /$ PBS) and left to react for $1 \mathrm{~h}$ at R.T. in saturated water vapours. Slides were washed for $3 \times 5$ min with PBS-T, $10 \mathrm{~s}$ in DI water and dried.

Quantification of uPA in cytosolic extracts from breast tumour tissue on antibody microarrays

Anti-uPA antibody (from Thermo Scientific) was spotted at 6.6 $\mu \mathrm{M}$ in PBS $1 \mathrm{X}$ buffer $(\mathrm{pH}=7.4)$ on $\mathrm{COOH}$, NHS and chitosan functionalized microstructured glass slides with 14 replicates (as shown in Fig. 2). PBS 1X (Buffer) and streptavidin-F555 (0.01 mg/ $\mathrm{ml}$ ) were spotted as negative and quality controls, respectively. Then all incubations and washing steps were the same as previously described in optimization of antibody microarray conditions.

On each slide, 6 microwells were firstly incubated with uPA standard solutions, prepared from Femtelle kit, at six different concentrations $(0 \mathrm{ng} / \mathrm{ml}, 0.2 \mathrm{ng} / \mathrm{ml}, 0.5 \mathrm{ng} / \mathrm{ml}, 1 \mathrm{ng} / \mathrm{ml}, 1.5 \mathrm{ng} / \mathrm{ml}$ and $2 \mathrm{ng} / \mathrm{ml}$ ) to obtain standard curve; 2 microwells were incubated with PBS $1 \mathrm{X}$ ( $\mathrm{pH}$ 7.4) buffer for negative controls; 16 microwells were incubated with non-diluted cytosolic extracts; 16 microwells were incubated with diluted (in 4\% BSA/PBS-T 0.1\%) cytosolic ex-tracts for 2 times (samples $\mathrm{N}^{\circ} 1-11$ ) or for 5 times (samples $\mathrm{N}^{\circ} 12$ to 16 ).

After washing and drying between each step, microwells were incubated with biotinylated anti-uPA antibody (prepared from Femtelle kit) diluted 2 times in 4\% BSA/PBS-T 0.1\%, then with streptavidin-F555 (0.01 mg/ml diluted in 1\% BSA/PBS).

\section{Fluorescence scanning and data analysis}

After drying, slides were scanned with the Microarray scanner Innopsys (InnoScan 710 Mapix 2Go software package) at wavelengths of $532 \mathrm{~nm}$ with high laser power. Data mining was accomplished with Mapix 2Go software package (Innopsys). The fluorescence signal obtained for each antigen-antibody system was determined as the average of the median fluorescence signal of

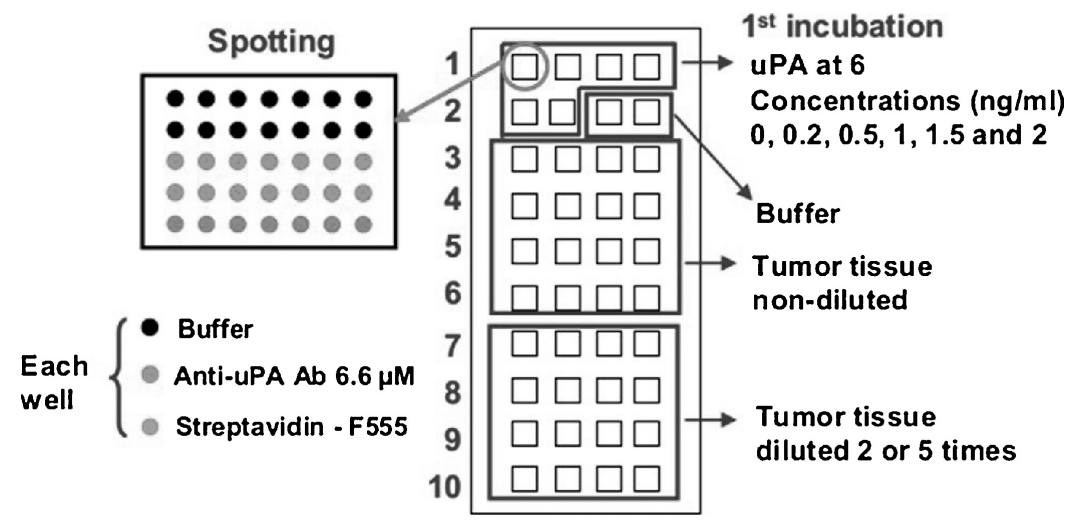

Fig. 2. Design of antibody microarray for the quantification of uPA in cytosolic extracts from breast tumour tissue. In each microwell, anti-uPA antibody was spotted in PBS $1 \mathrm{X}$ buffer at $6.6 \mu \mathrm{M}$ with 14 replications; PBS 1X (Buffer) and streptavidin-F555 (Strept-F555) were spotted as negative and quality controls, respectively. 6 microwells were incubated with uPA standard solutions at six different concentrations; 2 microwells with PBS 1 X buffer; 16 microwells with non-diluted cytosolic extracts; 16 microwells with cytosolic extracts diluted $1: 2$ (samples $\mathrm{N}^{\circ} 1-11$ ) or $1: 5$ (samples $\mathrm{N}^{\circ} 12-16$ ). 
several replicates. The signal-to-noise ratio (SNR) was the fluorescence signal of each antigen-antibody system divided by the signal of buffer spots (negative control). The statistical analyses were performed using $\mathrm{R}$ (version 3.0.0).

\section{Results and discussion}

Validation of biological activity of capture anti-uPA antibody

We firstly evaluated the biological activity of two capture antiuPA antibodies (from Santa Cruz Biotech and Thermo Scientific) by ELISA and compared the results with those obtained from Femtelle kit. Results presented in Fig. 3 indicated that both anti-uPA antibodies tested displayed good biological activity for detecting uPA standard solutions in ELISA and were validated and suitable for further study. However, their biological recognition towards uPA appeared less efficient compared to capture anti-uPA antibody from Femtelle kit. Indeed, in commercial ELISA kit, antibody/ antigen affinity is usually very high since the process is optimized to get best sensitivity and specificity of the assay.

\section{Optimization of antibody microarray conditions}

The implementation of efficient antibody microarrays requires optimizing important parameters such as surface chemistry, concentration of capture antibody and detection antibody. The influence of these factors is presented in the following.

\section{Influence of surface chemistry}

Anti-uPA antibody from Santa Cruz Biotech was chosen to evaluate the influence of surface chemistry. Results are shown in Fig. 4. As can be seen, capture anti-uPA antibody immobilized on APDMES, CMD and MAMVE surfaces could not sensitively detected uPA even at the highest concentration tested $(2 \mathrm{ng} / \mathrm{ml})$. In contrast, the immobilization of capture anti-uPA antibody on $\mathrm{COOH}$, NHS and chitosan surfaces allowed sensitive detection of uPA. Moreover, fluorescence intensities increased with the increasing concentration of uPA in the range tested, displaying good dynamic range for analysis.

The different surface chemistries tested allowed the immobilization of proteins through different interactions and environments. Among them, $\mathrm{COOH}$ and APDMES surfaces are monolayer functionalized surfaces allowing immobilization of proteins through non covalent binding (physisorption). NHS surface, a monolayer surface derived from the activation of $\mathrm{COOH}$ surface, allows covalent binding of proteins. Chitosan surface is a multilayer polysaccharide surface interacting with proteins

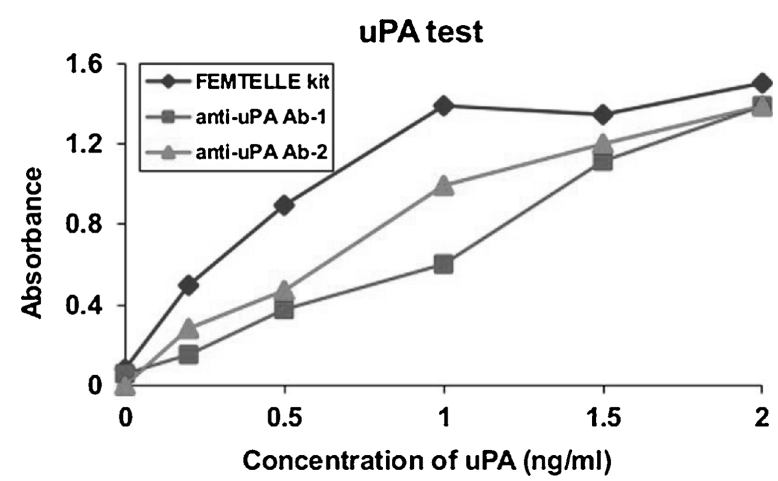

Fig. 3. Detection of uPA standard solutions by ELISA from Femtelle kit, using capture anti-uPA Ab-1 (from Santa Cruz Biotech) and capture anti-uPA Ab-2 (from Thermo Scientific).

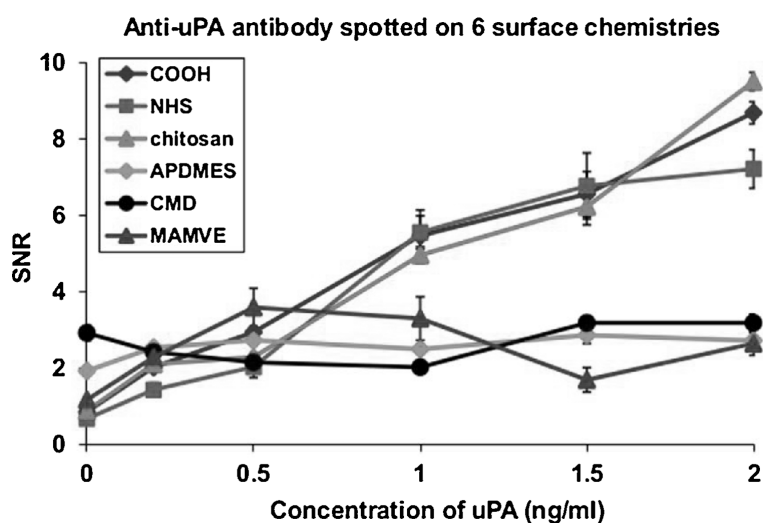

Fig. 4. uPA detection (SNR) with capture anti-uPA antibody (from Santa Cruz Biotech, spotted at $2.5 \mu \mathrm{M}$ ) immobilized on $\mathrm{COOH}$, NHS, chitosan, APDMES, CMD and MAMVE surfaces, as a function of uPA concentrations.

through physisorption. NHS-activated CMD and MAMVE surfaces are multi-layer surfaces, polysaccharide and synthetic polymer respectively, allowing covalent binding of proteins (Yang et al., 2012a). Our previous results showed that the efficiency of protein immobilization and consequently the maintaining of its biological activity is greatly affected by the physico-chemical characteristics of the surface. For example, the results of Yang et al. (2012a) showed that covalent immobilization (on NHS, CMD and MAMVE surfaces) of capture antibodies was more efficient than physical adsorption (on $\mathrm{COOH}$ surface) to retain biological activity of some immobilized proteins. In another study by Yang et al. (2013), we found that the immobilization of capture antibodies against 4 colorectal cancer (CRC) markers was less efficient on APDMES surface than on chitosan surface. Due to the unique structure of each protein, it is important to find its optimal surface. Therefore, $\mathrm{COOH}, \mathrm{NHS}$ and chitosan surfaces were selected for further immobilization of anti-uPA antibody.

\section{Influence of captured and detection anti-uPA antibodies concentrations}

Both anti-uPA antibody (from Santa Cruz Biotech and Thermo Scientific) were immobilized on $\mathrm{COOH}$, NHS and chitosan surfaces at different concentrations: $0.33 \mu \mathrm{M}, 0.67 \mu \mathrm{M}$ and $2.5 \mu \mathrm{M}$ for the former, and $3 \mu \mathrm{M}$ and $6.6 \mu \mathrm{M}$ for the latter. The choice of concentration was based on the maximal commercially available concentration of the antibody. Fig. 5 showed the influence of spotting concentration of capture anti-uPA antibodies.

As can be seen, SNR increased with the increasing of uPA concentrations as well as with spotting anti-uPA antibody concentrations on all three surfaces. Low concentration of capture anti-uPA antibody $(0.33 \mu \mathrm{M}$ and $0.67 \mu \mathrm{M})$ could hardly detect uPA. Moreover, on $\mathrm{COOH}$ surface, for very close spotting concentrations, immobilized anti-uPA Ab from Thermo Scientific (at $3 \mu \mathrm{M}$ ) showed much higher biological activity than immobilized anti-uPA Ab from Santa Cruz Biotech (at $2.5 \mu \mathrm{M}$ ). However, on NHS surface, both antibodies displayed similar level of biological activity for both spotting concentrations. On chitosan surface, anti-uPA Ab from Thermo Scientific could only detect uPA under high spotting concentration (at $6.6 \mu \mathrm{M}$ ) and it showed much lower biological activity than anti-uPA Ab from Santa Cruz Biotech (at $2.5 \mu \mathrm{M}$ ). This difference might be due to the fact that antibodies from different suppliers recognized different epitope of antigens.

Our previous study also showed that high spotting $\mathrm{Ab}$ concentration is necessary. For example, we found that spotting concentrations of capture antibody lower than $1 \mu \mathrm{M}$ could not allow the sensitive detection of corresponding antigens (Yang et al. 

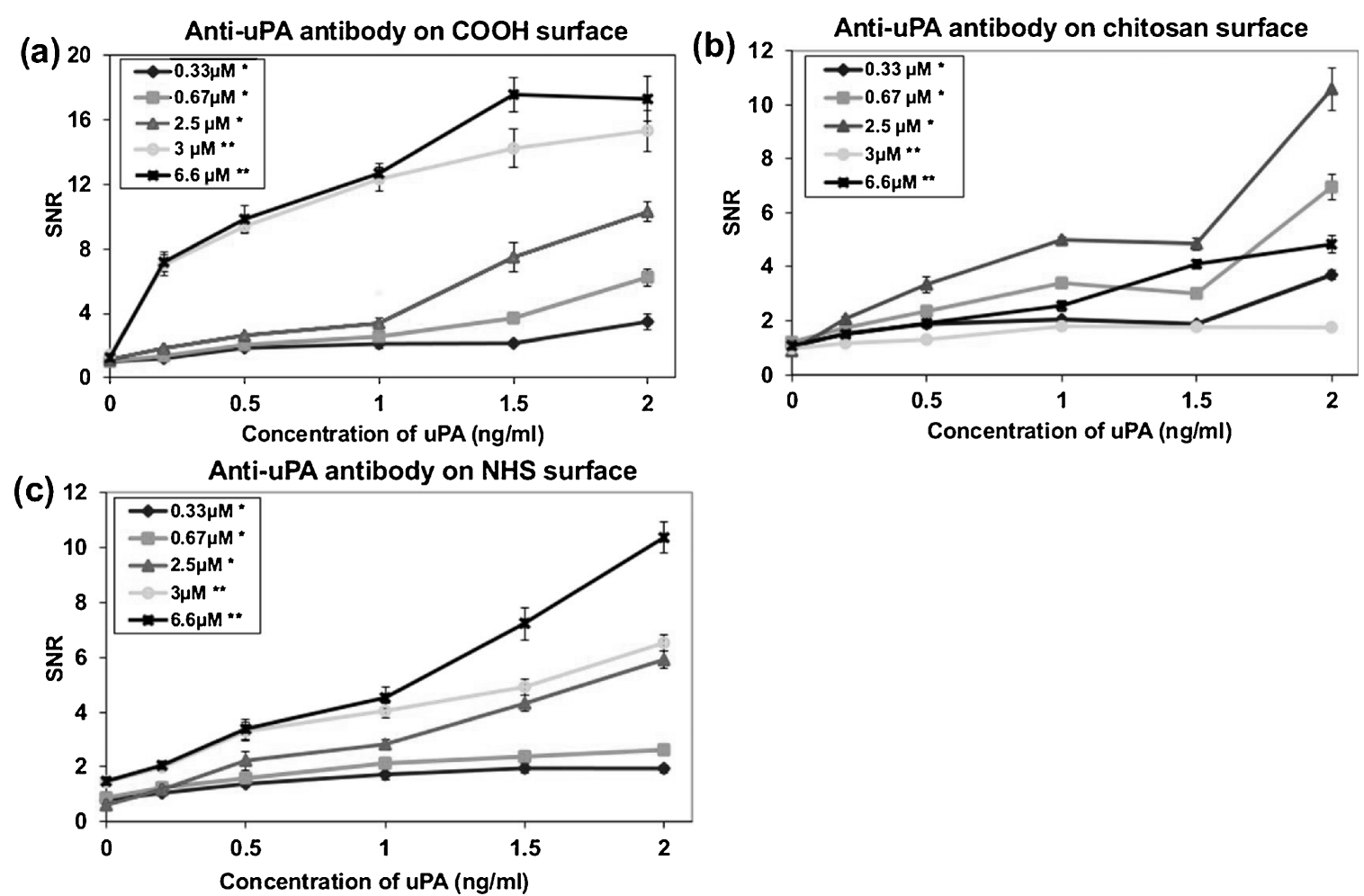

Fig. 5. Comparison of different spotting concentrations of anti-uPA antibodies for the detection of uPA standard solutions (from Femtelle kit) on COOH surface (a), chitosan surface (b) and NHS surface (c); * anti-uPA antibody from Santa Cruz Biotech was spotted at $0.33 \mu \mathrm{M}, 0.67 \mu \mathrm{M}$ and $2.5 \mu \mathrm{M}$; ** anti-uPA antibody from Thermo Scientific was spotted at $3 \mu \mathrm{M}$ and $6.6 \mu \mathrm{M}$.

2012a). Another study showed that $10 \mu \mathrm{M}$ capture antibody achieved the best results (Yang et al., 2013). Here, the stocking concentration of anti-uPA Ab from Santa Cruz Biotech is at $2.5 \mu \mathrm{M}$, which could be a limitation for the efficient detection of low concentration of uPA. Therefore, only anti-uPA Ab from Thermo Scientific was used for further study.

The concentration of detection anti-uPA antibody was also evaluated. Indeed, biotinylated antibody against uPA was obtained from Femtelle kit and no information about its concentration was given by the supplier. Thus, non-diluted and diluted (1:2 in 4\% BSA/ PBS-T $0.1 \%$ solution) biotinylated anti-uPA antibody were tested for the detection of capture anti-uPA antibody/uPA interaction. Results showed no significant difference between these two conditions on $\mathrm{COOH}$ and chitosan surfaces, whereas on NHS surface, diluted biotinylated antibody displayed the best performances (see Fig. S1). Indeed, high fluorescent signal was observed on NHS surface when non-diluted biotinylated anti-uPA antibody was used. Therefore, diluted (1:2) biotinylated anti-uPA antibody was chosen to quantify uPA concentration in breast tumour tissue extracts.

Fig. S1 compared the results obtained from diluted $(2 \times)$ or not diluted biotinylated anti-uPA detection antibody when capture anti-uPA antibody (from Santa Cruz Biotech) was spotted at $2.5 \mu \mathrm{M}$ on $\mathrm{COOH}$, NHS and chitosan surfaces. Results showed that no significant difference was observed between the two conditions on $\mathrm{COOH}$ and chitosan surfaces. In contrast, on NHS surface, diluted biotinylated anti-uPA antibody displayed better dynamic range for uPA detection than not diluted detection antibody which reached a

Table 1

Concentrations of uPA in 16 cytosolic extracts prepared from frozen breast tumour tissue samples.

\begin{tabular}{|c|c|c|c|c|c|c|}
\hline $\begin{array}{l}\text { No. of } \\
\text { sample }\end{array}$ & $\begin{array}{l}\text { Sample } \\
\text { weight } \\
(\mathrm{mg})\end{array}$ & $\begin{array}{l}\text { Total protein } \\
\text { concentration } \\
(\mathrm{mg} / \mathrm{ml})\end{array}$ & $\begin{array}{l}\text { uPA concentration by } \\
\text { Femtelle ELISA (ng/ml) }\end{array}$ & $\begin{array}{l}\text { uPA concentration by } \\
\text { Femtelle ELISA (ng/mg) }\end{array}$ & $\begin{array}{l}\text { uPA concentration by antibody } \\
\text { microarray }(\mathrm{ng} / \mathrm{ml})\end{array}$ & $\begin{array}{l}\text { uPA concentration by antibody } \\
\text { microarray }(\mathrm{ng} / \mathrm{mg})\end{array}$ \\
\hline 1 & 36 & 2.2 & 0.4 & 0.2 & 0.6 & 0.3 \\
\hline 2 & 39 & 1.6 & 0.9 & 0.6 & 0.8 & 0.5 \\
\hline 3 & 43 & 2.3 & 1.4 & 0.6 & 1.8 & 0.8 \\
\hline 4 & 54 & 2.1 & 1.5 & 0.7 & 1.4 & 0.6 \\
\hline 5 & 223 & 3.7 & 2.1 & 0.6 & 2.9 & 0.8 \\
\hline 6 & 74 & 3.5 & 2.3 & 0.7 & 2.5 & 0.7 \\
\hline 7 & 320 & 3.8 & 2.4 & 0.6 & 3.1 & 0.8 \\
\hline 8 & 41 & 1.2 & 3.1 & 2.6 & 2.2 & 1.8 \\
\hline 9 & 36 & 1.7 & 3.3 & 1.9 & 1.6 & 0.9 \\
\hline 10 & 110 & 4.0 & 3.6 & 0.9 & 4.6 & 1.2 \\
\hline 11 & 44 & 2.7 & 3.9 & 1.4 & 3.3 & 1.2 \\
\hline 12 & 115 & 4.5 & 4.3 & 1.0 & 7.2 & 1.6 \\
\hline 13 & 51 & 2.4 & 4.6 & 1.9 & 6.1 & 2.5 \\
\hline 14 & 115 & 3.9 & 6.4 & 1.6 & 8.6 & 2.2 \\
\hline 15 & 53 & 3.8 & 7 & 1.8 & 5.9 & 1.6 \\
\hline 16 & 78 & 2.4 & 8 & 3.3 & 8.4 & 3.4 \\
\hline
\end{tabular}


plateau value at $1.5 \mathrm{ng} / \mathrm{ml}$ of uPA. Therefore, diluted $(1: 2)$ biotinylated anti-uPA antibody was chosen to quantify uPA concentration in tumor tissue extracts.

To summarize, the optimal conditions for the detection of uPA using our customized antibody microarray were defined as following: (1) three surface chemistries ( $\mathrm{COOH}$, NHS and chitosan surfaces) were selected; (2) anti-uPA antibody from Thermo Scientific was chosen as capture antibody and was spotted at highest concentration (6.6 $\mu \mathrm{M})$; (3) biotinylated anti-uPA antibody was used in dilution $(1: 2)$.

\section{Quantification of UPA from breast tumour tissue extracts}

16 cytosolic extracts from frozen breast tumour tissue were evaluated for uPA quantification using our optimized antibody microarray and results were compared to data obtained by Femtelle ELISA kit. Among three surfaces used, high background was observed on chitosan and NHS surfaces for cytosolic extracts incubation, making data analysis impossible. High background was probably due to unspecific binding of proteins contained in cytosolic extracts. In contract, Ab microarray with $\mathrm{COOH}$ surface had low unspecific binding in the presence of cytosolic extracts, which were therefore analysed and compared with ELISA kit (Table 1).

We firstly used Spearman correlation coefficients to analyse the possible relationships between the two methods. The $\mathrm{r}$ obtained was high, equal to 0.9 ( $p=0.00004,95 \%$ CI was $0.73-0.97)$, suggesting that there was a very strong positive correlation between these two methods.

Then we further examined the agreement and correlation of the results obtained by these two methods by using Bland-Altman difference plots and Passing and Bablok, shown in Fig. 6a and b, respectively. From Bland-Altman difference plots analysis (Fig. 6a), the mean of relative difference is $35.7 \%$, suggesting that the difference between the two methods was low. Furthermore, the correlation between difference and mean was 0.16 ( $p=0.55,95 \% \mathrm{CI}$ was -0.36 to 0.61 ), indicating that the difference slightly increased with the magnitude of the measurement, but the relation was very week $(p=0.55)$. Fig. $6 \mathrm{~b}$ showed the linear regression obtained from Passing and Bablok as well as its 95\% confidence intervals (CI) (95\% $\mathrm{CI}=0.89-1.56)$. The regression line between the two methods was Ab microarray $=1.31$ X ELISA-0.23. As shown in Fig. $6 \mathrm{~b}$, the majority plots are in the $95 \% \mathrm{CI}$ range, indicating that uPA concentration obtained from our antibody microarray was consistent with those obtained from ELISA.

The clinical relevance of uPA is based on its concentration in total protein content. Low levels of uPA ( $\leq 3 \mathrm{ng} / \mathrm{mg}$ of total protein) are associated with low risk of recurrence and may have few or no benefit from adjuvant chemotherapy. We therefore calculated uPA concentration in total protein content and compared with ELISA kit. Based on ELISA results, only one sample (No. 16) displayed higher concentration than $3 \mathrm{ng} / \mathrm{mg}$. Our results also showed that except for sample 16, all samples displayed uPA concentrations less than $3 \mathrm{ng} / \mathrm{mg}$ (Table 1 ).

We compared the performance of our antibody microarray with the study of Weissenstein et al. (2006) based on several aspects. 1) For sample volume required, our antibody microarray consumed more than 10 times less samples compared with them, which could further reduce sample consumption; 2) Both studies obtained high $r$ value from Spearman correlation coefficients analysis, ( $r$ in our study was slightly lower, 0.9 vs. 0.97 ), suggesting that there was a very strong positive correlation between antibody microarray and ELISA in both studies; 3) From Bland-Altman analysis, we can see that the mean difference between antibody microarray and ELISA in our study was slightly higher (35.7\% vs. 29.4\%). However, the correlation between difference and average was lower in our study (0.16 vs. 0.25$)$, which means that the difference was less dependent on the value of measurement in our study; 4) The slope of regression line in our study was closer to 1 (1.31 vs. 0.56), meaning that the results obtained by our antibody microarray was closer to ELISA; 5) In terms of clinical relevance, in Weissenstein et al. (2006), 2\% of samples whose uPA concentration in total protein content were wrongly classified. In comparison, in our studies, all samples could be correctly classified. Therefore, our study showed better performance than the one of Weissenstein et al. (2006) while less samples were included in our study (16 vs. 50).

Although our customized antibody microarray show high promises for uPA quantification, our studies have its own limitations. Firstly, our sample size is small and only 16 samples were tested, further large scale investigation is needed to validate its performance. Secondly, we used frozen breast tumour tissue samples instead of FFPE tissue, among which the latter is the main source of patient's sample worldwide. We therefore anticipate to test FFPE tissue in the future.

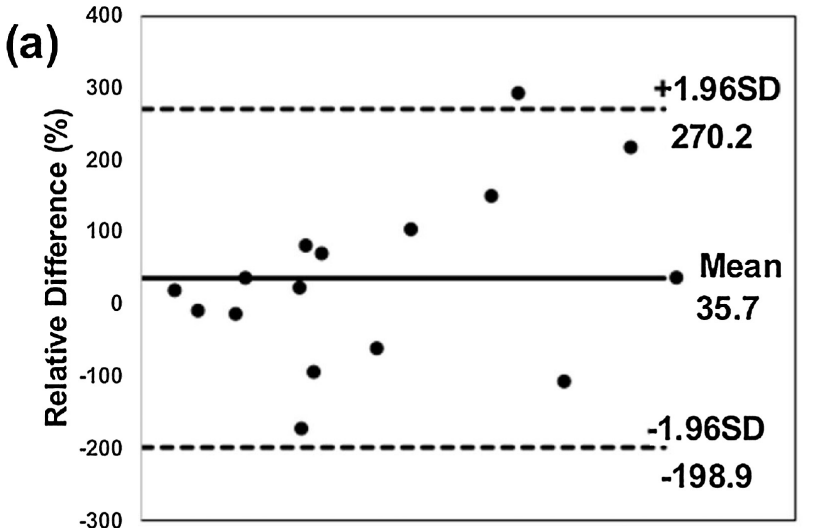

Average of ELISA and Ab microarray

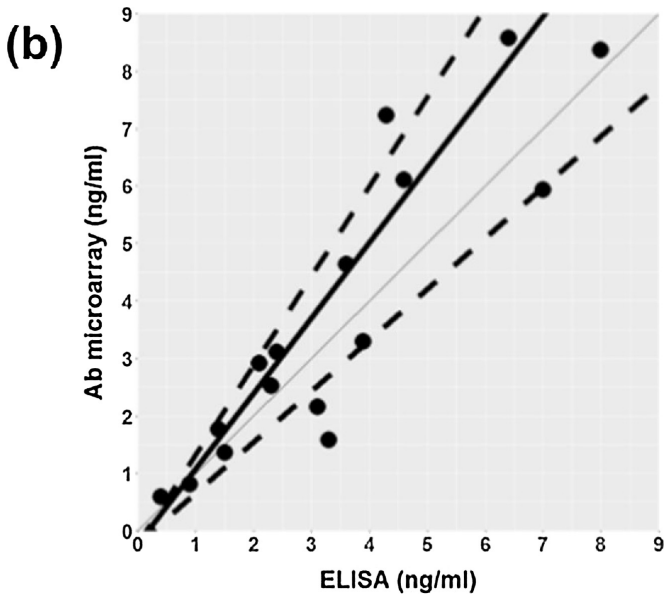

Fig. 6. Agreement (a) and correlation (b) of the results obtained by antibody microarray and ELISA by using Bland-Altman difference plots and Passing and Bablok analysis, respectively. 


\section{Conclusion}

In conclusion, the performance of antibody microarray is influenced by many parameters such as surface chemistry, concentration of captured and detection antibodies. In this study, we firstly optimized these factors and found three surfaces $(\mathrm{COOH}$, NHS and chitosan) could retain the biological activity of captured Ab. Moreover, high spotting concentration of captured Ab was necessary. Then under optimal condition, we assessed uPA concentration in 16 cytosolic extracts from breast tumor tissues. Our customized antibody microarray could correctly classify uPA concentration in total protein content. Furthermore, unlike Femtelle ${ }^{\circledR}$ ELISA kit requires $100 \mu$ l of tumour tissue extraction solution for incubation, only $1 \mu \mathrm{l}$ of this solution is needed with our antibody microarray. Therefore, our method is an improvement to traditional ELISA and it is a powerful and promising tool for analysing biological molecules in miniaturization form.

\section{Conflict of interests}

The authors declare no conflict of interests.

\section{Acknowledgements}

China Scholarship Council is grateful to offer Ph.D. fellowship to Liu SHI. The CNANO Rhône-Alpes and BQR Ecole Centrale de Lyon are acknowledged for financial support. NanoLyon is acknowledged for technical support.

\section{Appendix A. Supplementary data}

Supplementary data associated with this article can be found, in the online version, at https://doi.org/10.1016/j.jab.2018.01.001.

\section{References}

Balboni, I., Limb, C., Tenenbaum, J.D., Utz, P.J., 2008. Evaluation of microarray surfaces and arraying parameters for autoantibody profiling. Proteom 8, 3443 3449.

Becker, K.F., Schott, C., Hipp, S., Metzger, V., Porschewski, P., Beck, R., et al., 2007. Quantitative protein analysis from formalin-fixed tissues: implications for translational clinical research and nanoscale molecular diagnosis. J. Pathol. 211 370-378.

Cretich, M., Damin, F., Chiari, M., 2014. Protein microarray technology: how far off is routine diagnostics? Analyst 139, 528-542.

Duffy, M.J., McGowan, P.M., Harbeck, N., Thomssen, C., Schmitt, M., 2014. uPA and PAI-1 as biomarkers in breast cancer: validated for clinical use in level-ofevidence-1 studies. Breast Cancer Res. 16, 428.

Harbeck, N., Kates, R.E., Look, M.P., Meijer-Van Gelder, M.E., Klijn, J.G., Kruger, A., et al., 2002. Enhanced benefit from adjuvant chemotherapy in breast cancer patients classified high-risk according to urokinase-type plasminogen activator (uPA) and plasminogen activator inhibitor type $1(\mathrm{n}=3424)$. Cancer Res. 62 , 4617-4622.

Harbeck, N., Schmitt, M., Meisner, C., Friedel, C., Untch, M., Schmidt, M., et al., 2013. Ten-year analysis of the prospective multicentre Chemo-N0 trial validates American Society of Clinical Oncology (ASCO)-recommended biomarkers uPA and PAI-1 for therapy decision making in node-negative breast cancer patients. Eur. J. Cancer 49, 1825-1835.

Harris, L.N., Ismaila, N., McShane, L.M., Andre, F., Collyar, D.E., Gonzalez-Angulo, A. M., et al., 2016. Use of biomarkers to guide decisions on adjuvant systemic therapy for women with early-stage invasive breast cancer: American society of clinical oncology clinical practice guideline. J. Clin. Oncol. 34, 1134-1150.

Hu, S., Xie, Z., Qian, J., Blackshaw, S., Zhu, H., 2012. Functional protein microarray technology. Wiley Interdiscip. Rev. Syst. Biol. Med. 3, 255-268.

Janicke, F., Prechtl, A., Thomssen, C., Harbeck, N., Meisner, C., Untch, M., et al., 2001. Randomized adjuvant chemotherapy trial in high-risk, lymph node-negative breast cancer patients identified by urokinase-type plasminogen activator and plasminogen activator inhibitor type 1. J. Natl. Cancer Inst. 93, 913-920.

Kwaan, H.C., Mazar, A.P., McMahon, B.J., 2013. The apparent uPA/PAI-1 paradox in cancer: more than meets the eye. Semin. Thromb. Hemost. 39, 382-391.

Look, M.P., van Putten, W.L., Duffy, M.J., Harbeck, N., Christensen, I.J., Thomssen, C., et al., 2002. Pooled analysis of prognostic impact of urokinase-type plasminogen activator and its inhibitor PAI-1 in 8377 breast cancer patients. J. Natl. Cancer Inst. 94, 116-128.

Malinowsky, K., Bollner, C., Hipp, S., Berg, D., Schmitt, M., Becker, K.F., 2010. UPA and PAI-1 analysis from fixed tissues - new perspectives for a known set of predictive markers. Curr. Med. Chem. 17, 4370-4377.

Mazurczyk, R., El Khoury, G., Dugas, V., Hannes, B., Laurenceau, E., Cabrera, M., Chevolot, Y., 2008. Low-cost, fast prototyping method of fabrication of the microreactor devices in soda-lime glass. Sens. Actuators B: Chem. 128, 552-559.

McMahon, B., Kwaan, H.C., 2008. The plasminogen activator system and cancer. Pathophysiol. Haemost. Thromb. 36, 184-194.

Schueller, G., Jaromi, S., Ponhold, L., Fuchsjaeger, M., Memarsadeghi, M., Rudas, M., et al., 2008. US-guided 14-gauge core-needle breast biopsy: results of a validation study in 1352 cases. Radiology 248, 406-413.

Shukla, S., Govekar, R.B., Sirdeshmukh, R., Sundaram, C.S., D’Cruz, A.K., Pathak, K.A., et al., 2007. Tumor antigens eliciting autoantibody response in cancer of gingivo-buccal complex. Proteom. Clin. Appl. 1, 1592-1604.

Sutandy, F.X., Qian, J., Chen, C.S., Zhu, H., 2013. Overview of protein microarrays. Curr. Protoc. Protein Sci. (Chapter 27, Unit 27.1.).

Weissenstein, U., Schneider, M.J., Pawlak, M., Cicenas, J., Eppenberger-Castori, S. Oroszlan, P., et al., 2006. Protein chip based miniaturized assay for the simultaneous quantitative monitoring of cancer biomarkers in tissue extracts. Proteom 6, 1427-1436.

Wilson, R., Kavia, S., 2009. Comparison of large-core vacuum-assisted breast biopsy and excision systems. Recent Results Cancer Res. 173, 23-41.

Yang, Z., Chevolot, Y., Ataman-Önal, Y., Choquet-Kastylevsky, G., Souteyrand, E., Laurenceau, E., 2012a. Cancer biomarkers detection using 3D microstructured protein chip: implementation of customized multiplex immunoassay. Sens. Actuators B: Chem. 175, 22-28.

Yang, Z., Chevolot, Y., Gehin, T., Solassol, J., Mange, A., Souteyrand, E., Laurenceau, E. 2012b. Improvement of protein immobilization for the elaboration of tumourassociated antigen microarrays: application to the sensitive and specific detection of tumour markers from breast cancer sera. Biosens. Bioelectron. 40, 385-392.

Yang, Z., Chevolot, Y., Gehin, T., Dugas, V., Xanthopoulos, N., Laporte, V., et al., 2013. Characterization of three amino-functionalized surfaces and evaluation of antibody immobilization for the multiplex detection of tumour markers involved in colorectal cancer. Langmuir 29, 1498-1509. 